\title{
Intraband exciton relaxation in a biased lattice with long-range correlated disorder
}

\author{
E. Díaz and F. Domínguez-Adame \\ Departamento de Física de Materiales, GISC, Universidad Complutense, E-28040 Madrid, Spain \\ (Received 10 December 2007; revised manuscript received 23 February 2008; published 1 April 2008)
}

\begin{abstract}
We numerically study the intraband exciton relaxation in a one-dimensional lattice with a scale-free disorder in the presence of a linear bias. Exciton transport is the incoherent hopping over the eigenstates of the static lattice. The site potential of the unbiased lattice is long-range-correlated with a power-law spectral density $S(k) \sim 1 / k^{\alpha}, \alpha>0$. The lattice supports a phase of extended states at the center of the band, provided $\alpha$ is larger than a critical value $\alpha_{c}$ [F. A. B. F. de Moura and M. L. Lyra, Phys. Rev. Lett. 81, 3735 (1998)]. When the bias is applied, the absorption spectrum displays clear signatures of the Wannier-Stark ladder [E. Díaz, F. Domínguez-Adame, Yu. A. Kosevich, and V. A. Malyshev, Phys. Rev. B 73, 174210 (2006)]. We demonstrate that in unbiased lattices and in weakly correlated potentials, the decay law is nonexponential. However, the decay is purely exponential when the bias increases and $\alpha$ is large. We relate this exponential decay to the occurrence of the Wannier-Stark ladder in the exciton band.
\end{abstract}

DOI: $10.1103 /$ PhysRevB.77.134201

PACS number(s): 32.50.+d, 71.35.Aa, 78.30.Ly

\section{INTRODUCTION}

Long-range correlations with spectral densities of the form $S(k) \sim 1 / k^{\alpha}$ often arise in nature. ${ }^{1,2}$ It has been argued that long-range correlations in nucleotide sequences can explain the long-distance charge transport in DNA. ${ }^{3-6}$ This type of correlation may result in a phase of extended states at the center of the band, provided $\alpha$ is larger than a critical value $\alpha_{c}{ }^{7}$ Therefore, a localization-delocalization transition at the two mobility edges, separating the phase of extended states at the band center from the localized ones at the band tails, is found in these systems. ${ }^{8}{ }^{89}$ It was demonstrated later that $\alpha_{c}$ $=2$ is the universal critical value for the localizationdelocalization transition to occur in the model introduced in Ref. 7, which is independent of the magnitude of disorder. ${ }^{10}$

Bloch-like oscillations ${ }^{11}$ of quasiparticles are known to arise in biased one-dimensional (1D) systems with $S(k)$ $\sim 1 / k^{\alpha}$ spectral density. ${ }^{12}$ These oscillations provide a way to measure the energy width of the delocalized phase since it determines the amplitude of the oscillations. In addition, the frequency-domain counterpart of the Bloch oscillations, the so-called Wannier-Stark ladder (WSL) ${ }^{13}$ was also observed in the optical absorption spectrum, in spite of the underlying randomness. ${ }^{14}$ Strong correlations in the disorder facilitate the observation of the WSL. At $\alpha>\alpha_{c}$, when the phase of extended states emerges at the center of the band, a periodic series of identical peaks is found to build up at the center of the absorption band. This periodic pattern was related to the Wannier-Stark quantization of the energy spectrum in the disordered lattice. ${ }^{14}$

In this work, we report further progress along this line. We present a model Hamiltonian for excitons interacting with vibrations of the host medium. We assume that there exists an intrinsic bias affecting the exciton dynamics as, for instance, that which occurs in dendrimers. ${ }^{15}$ Furthermore, site energies are assumed to be long-range-correlated with $S(k) \sim 1 / k^{\alpha}$ spectral density. The aim of this work is twofold. First, we analyze how intraband relaxation, due to the coupling to the vibrations of the glassy host medium, affects the time behavior of the exciton decay in the biased lattice. Sec- ond, we look for signatures of the WSL in the radiative decay of excitons.

The outline of the paper is as follows. In Sec. II, we present our model, which is based on a tight-binding Hamiltonian of an exciton in a long-range-correlated potential landscape, which is subjected to a linear bias. In Sec. III, we recall the basic physics of the exciton intraband relaxation due to the coupling to host vibrations. Exciton dynamics will be described by a Pauli master equation for the populations of the exciton states. ${ }^{16}$ The central parts of the paper are Secs. IV and V, where we present the results of numerical simulations of the time decay of fluorescence after broadband pulse excitation in disorder-correlated systems. We discuss its dependence on the driving parameters of the model (bias magnitude, disorder strength, and correlation exponent $\alpha$ ) in detail and provide evidence of what the WSL reveals in the intraband exciton relaxation. We find that the fluorescence exponentially decays when localization by the bias prevails over localization by a disorder. We relate this exponential decay to the occurrence of the WSL. When the disorder is large enough or long-range correlations are weak $\left(\alpha<\alpha_{c}\right)$, the time dependence of the fluorescence decay is nonexponential. Finite size effects on the time decay of fluorescence are analyzed in Sec. VI. Finally, Sec. VII concludes the paper.

\section{MODEL HAMILTONIAN}

We consider a biased tight-binding model with diagonal disorder on an otherwise regular 1D open lattice of spacing unity and $N$ sites ( $N$ is assumed to be even). We assign two levels to each lattice site, ground and excited, and consider optical transitions between them with transition energy $\mathcal{E}_{n}$ $=\overline{\mathcal{E}}+\varepsilon_{n}$. The stochastic part $\varepsilon_{n}$ is generated according to ${ }^{7}$

$$
\varepsilon_{n}=\sigma C_{\alpha} \sum_{k=1}^{N / 2} \frac{1}{k^{\alpha / 2}} \cos \left(\frac{2 \pi k n}{N}+\phi_{k}\right),
$$

where the normalization constant is given by 


$$
C_{\alpha}=\sqrt{2}\left(\sum_{k=1}^{N / 2} \frac{1}{k^{\alpha}}\right)^{-1 / 2}
$$

Here, $\phi_{1}, \ldots, \phi_{N / 2}$ are uncorrelated random phases uniformly distributed within the interval $[0,2 \pi]$. The distribution (1a) and (1b) has a zero mean $\left\langle\varepsilon_{n}\right\rangle=0$ and standard deviation $\left\langle\varepsilon_{n}^{2}\right\rangle^{1 / 2}=\sigma$, where $\langle\cdots\rangle$ indicates averaging over realizations of random phases $\phi_{k}$. The quantity $\sigma$ will be referred to as the magnitude of disorder.

The model Hamiltonian is

$$
\begin{aligned}
\mathcal{H}= & \sum_{n=1}^{N}\left[\mathcal{E}_{n}-U\left(n-\frac{N}{2}\right)\right]|n\rangle\langle n| \\
& -J \sum_{n=1}^{N-1}(|n\rangle\langle n+1|+| n+1\rangle\langle n|) .
\end{aligned}
$$

Here, $|n\rangle$ denotes the state in which the $n$th site is excited, whereas all the other sites are in the ground state. The term $-U(n-N / 2)$ describes the linear bias. The intersite transfer integrals in Eq. (2) are restricted to nearest neighbors and they are set to $-J$ over the entire lattice. Also, we set $\overline{\mathcal{E}}=0$ hereafter without loss of generality.

As we already mentioned in Sec. I, the unbiased model supports a phase of extended states at the center of the band, provided the correlation exponent $\alpha$ is larger than a critical value $\alpha_{c}$. On the contrary, all the states are localized at $\alpha$ $<\alpha_{c}$.

\section{INTRABAND RELAXATION}

Excitons can hop between the eigenstates of the Hamiltonian (2) only if coupling to vibrations is taken into account. We assume that this coupling is weak and do not consider polaron effects. The exciton-vibration interaction causes the incoherent hopping of excitons from one eigenstate to another. Only one-phonon processes are considered throughout the paper. We take the transition rate from the state $\psi_{\mu}$ (with energy $E_{\mu}$ ) to the state $\psi_{\nu}$ (with energy $E_{\nu}$ ) according to (see Ref. 16 for further details)

$$
W_{\mu \nu}=W_{0} S\left(\left|\Delta E_{\mu \nu}\right|\right) F\left(\Delta E_{\mu \nu}, T\right) \mathcal{I}_{\mu \nu},
$$

with $\Delta E_{\mu \nu} \equiv E_{\mu}-E_{\nu}$. The constant $W_{0}$ characterizes the amplitude of transitions. A spectral density function of the form $S(|\Delta E|)=|\Delta E| / J$ holds for glassy hosts. ${ }^{17,18}$ Temperature $T$ enters into this expression through the function $F(\Delta E, T)$ $=\theta(\Delta E)+n(\Delta E, T)$, where $\theta$ is the Heaviside step function and $n(\Delta E, T)=\left[\exp \left(|\Delta E| / k_{B} T\right)-1\right]^{-1}$ is the occupation number of the vibration mode with frequency $\Delta E / \hbar$. The parameter

$$
\mathcal{I}_{\mu \nu} \equiv \sum_{n=1}^{N} \psi_{\mu, n}^{2} \psi_{\nu, n}^{2}
$$

represents the overlap integral of exciton probabilities for the states $\psi_{\mu}$ and $\psi_{\nu}$. Notice that the transition rates meet the principle of detailed balance, namely $W_{\nu \mu}$ $=W_{\mu \nu} \exp \left(\Delta E_{\mu \nu} / k_{B} T\right)$.

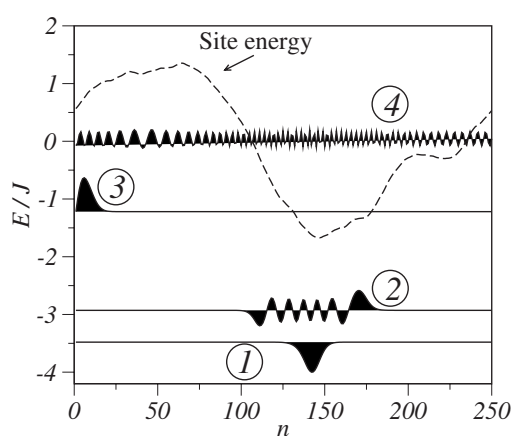

FIG. 1. A subset of eigenstates for a typical realization of the random energy potential (dashed line) for a lattice of size $N=250$, magnitude of disorder $\sigma=J$, and correlation exponent $\alpha=4$ (larger than the critical value $\alpha_{c}=2$ ). The baseline indicates the energy of the eigenstate. States 1 and 3 are those with larger oscillator strengths.

We describe the process of exciton relaxation by means of the Pauli master equation for the level population $P_{\mu}$ of the $\mu$ th exciton eigenstate,

$$
\frac{d P_{\mu}}{d t}=-\gamma_{\mu} P_{\mu}+\sum_{\nu=1}^{N}\left(W_{\nu \mu} P_{\nu}-W_{\mu \nu} P_{\mu}\right)
$$

where $\gamma_{\nu}=\gamma f_{\mu}$ is the spontaneous emission rate of the $\mu$ th exciton state, while $\gamma$ is that of a monomer, $f_{\mu}=\left(\sum_{n=1}^{N} \psi_{\mu, n}\right)^{2}$ being the dimensionless oscillator strength. After broadband pulse excitation, each level is populated according to its oscillator strength, namely, $P_{\mu}(0)=f_{\mu} / N$. Consequently, the initial total population is normalized to unity, $\Sigma_{\mu} P_{\mu}(0)=1$. The normalized fluorescence after pulse excitation is obtained from

$$
I(t)=N \frac{\sum_{\mu=1}^{N} f_{\mu} P_{\mu}(t)}{\sum_{\mu=1}^{N} f_{\mu}^{2}},
$$

which is the magnitude of interest in this work.

\section{UNBIASED SYSTEM}

We first discuss the exciton decay of the unbiased system $(U=0)$, which aims to separate the effects of bias from those related to the disordered nature of the model. We have numerically diagonalized the Hamiltonian (2) by means of standard methods and obtained the eigenstates $\psi_{\mu}$ and eigenvalues $E_{\mu}$ for different values of the correlation exponent $\alpha$ and magnitude of disorder $\sigma$, taking open linear lattices into account. In Fig. 1, we show a subset of eigenstates obtained for a typical random realization of the potential landscape for $\alpha=4$, larger than the critical value $\alpha_{c}=2$. The baselines display the corresponding eigenenergies. The lowest state in Fig. 1 (labeled 1) shows a bell-like shape and carries a large oscillator strength. There are several states of such type (not shown in Fig. 1), which are close in energy to state 1 and do not overlap with each other. On increasing the energy, one observes eigenstates, such as the one labeled 2 in Fig. 1, which are more extended, as compared to the lowest one, 


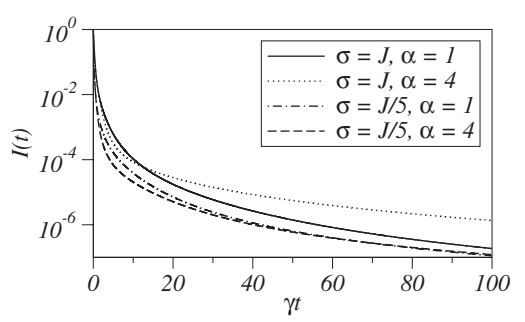

FIG. 2. Time dependence of the fluorescence decay after pulse excitation in weakly $(\alpha=1)$ and strongly $(\alpha=4)$ correlated 1D lattices when $U=0$, for two different magnitudes of disorder. The lattice size is $N=250$ and the results comprise the average over 200 realizations of the disorder.

and present several nodes within the localization segment. The oscillator strength of such states is much smaller (dark states). Remarkably, going further up in energy, we again find bell-like states, as the one labeled 3 in Fig. 1, which are characterized by a large oscillator strength. Finally, on approaching the center of the band, one expects the occurrence of extended states. State 4 in Fig. 1 represents an example.

The situation is different when $\alpha$ is smaller than the critical value $\alpha_{c}=2$. The level structure is then similar to what is found in the standard 1D Anderson model (uncorrelated disorder). Bell-like states lay at the bottom of the band and dark states, localized over larger segments, are higher in energy (see, e.g., Fig. 1 in Ref. 19). In addition, there are no extended states such as that labeled 4 in Fig. 1 at $\alpha<\alpha_{c}$.

Keeping in mind the different level structures for $\alpha$, which are larger and smaller than the critical value $\alpha_{c}$, we obtained the normalized fluorescence after pulse excitation (6). Hereafter, we restrict ourselves to $T=0$; we find that our main conclusions are valid when the temperature is a fraction of $J / k_{B}$. This can be understood from the large energy difference between states 1 and 3 in Fig. 1, which are those strongly coupled to the light due to their high oscillator strength. In other words, it is highly improbable that an initial excitation of state 1 can be transferred to state 3 after several hops, at least at low $T$. Also, we choose the parameter $W_{0}=\gamma$ hereafter, so that a radiative decay is more favorable than an intraband relaxation. This statement can be understood from previous estimations in unbiased lattices with uncorrelated disorder. ${ }^{20}$ When $\sigma=J$, the largest intraband transition rate $W_{\mu \nu}$ is of the order $\sim 0.06 W_{0}$, but the higher spontaneous emission rate $\gamma_{\mu}$ is about $\sim 9 \gamma$.

Figure 2 shows the fluorescence decay after pulse excitation as a function of $\gamma t$ for various set parameters and $W_{0}$ $=\gamma$. The lattice size is $N=250$ and the results comprise the average over 200 realizations of the disorder. In all cases, the decay is clearly nonexponential. At large times, the decay law fits a stretched exponential $I(t) \sim \exp \left[-\left(t / \tau_{\infty}\right)^{\beta}\right]$, where both $\tau_{\infty}$ and $\beta<1$ depend on the correlation exponent $\alpha$. The fluorescence curves are similar when the magnitude of disorder $\sigma$ is small, as can be seen in Fig. 2. However, on increasing the magnitude of disorder, the fluorescence decay is slower in strongly correlated systems.

The slowing down of the fluorescence decay can be understood as follows. When $\alpha$ is smaller than $\alpha_{c}$, high-energy states are weakly coupled to the light due to their low oscil- lator strength. The excitation is then transferred from the high-energy states (dark states) to those at the bottom of the band (bell-like states). The exciton is stuck in the bottom state and it emits a photon on average after time $1 / \gamma_{1}$ has elapsed. On the contrary, when $\alpha$ is larger than $\alpha_{c}$, the initial exciton population does not decrease monotonously on increasing energy due to the occurrence of bell-like states such as that labeled 3 in Fig. 1 (recall that the initial level population is proportional to the corresponding oscillator strength). In contrast to bell-like states at the bottom of the band, those highly populated states after excitation may not decay radiatively: an exciton that is initially located at state 3 in Fig. 1 may be transferred to a state of lower energy whose oscillator strength is vanishingly small (e.g., state 2 in Fig. 1). The exciton is then scattered to the bottom states (e.g., state 1 in Fig. 1) and then emits a photon. Consequently, on average, there are more intraband scattering events through dark states when $\alpha$ is large, yielding a slowdown, which is observed in Fig. 2.

\section{BIASED SYSTEM}

In disorder-free systems, switching the bias on results in a reorganization of the level structure, which becomes equally spaced with level spacing $U .{ }^{13}$ The corresponding eigenstates become localized within a localization segment of length $L_{U}=W / U$ in units of the spatial period, where $W$ is the bandwidth. In $1 \mathrm{D}$ disorder-free systems, this bandwidth is $W$ $=4 J$. This structure is revealed in photoluminescence ${ }^{21,22}$ and photoconductivity $^{23}$ spectra as a series of equally spaced peaks. Deviations from perfect periodicity broaden the peaks and make them unresolved when disorder is uncorrelated and large. As mentioned in Sec. I, long-range correlations facilitate the occurrence of the WSL even if disorder is large. ${ }^{14} \mathrm{In}$ this case, a periodic pattern is found to build up at the center of the optical absorption band, where the absorption spectrum line shape is obtained from

$$
A(E)=\frac{1}{N}\left\langle\sum_{\mu=1}^{N} f_{\mu} \delta\left(E-E_{\mu}\right)\right\rangle .
$$

The period of the periodic pattern is equal to $U$, as for the WSL in an ideal lattice, and is independent of the system size $N$. This pattern was attributed to the Wannier-Stark quantization of the energy spectrum in the long-range-correlated disordered lattice. ${ }^{14}$

In Fig. 3, we plotted the absorption spectra calculated for disordered biased chains of $N=250$ sites, choosing the magnitude of disorder $\sigma=J$ and the bias magnitude $U=0.05 J$. Averages over $10^{6}$ realizations of disorder were performed in Eq. (7). Two values of the correlation exponents were considered, $\alpha=1$ and $\alpha=4$. The spectra broaden as compared to those of the unbiased systems (not shown in the figure). In Ref. 14, we proved that the spectrum broadens when $\sigma^{*}$ $<U N$, namely, when the absorption linewidth $\sigma^{*}$ in the unbiased system is smaller than the potential energy drop across the entire lattice $U N$. The width of the absorption spectrum (full width at half maximum) when $U$ is low is of the order of $U N=12.5$, as observed in Fig. 3. However, the 


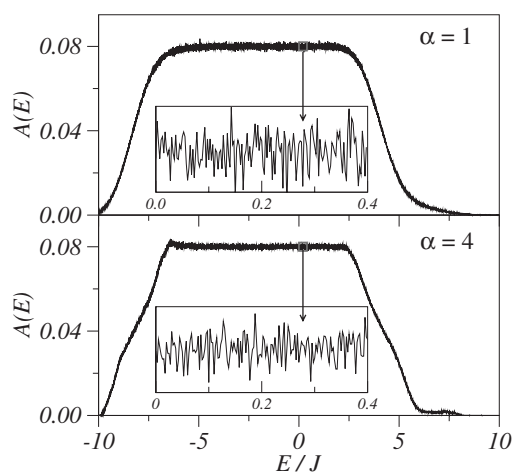

FIG. 3. Absorption spectra of biased chains $(U=0.05 J)$ with $N$ $=250$ sites calculated for two values of the correlation exponent $\alpha$, shown in the plot. The magnitude of disorder is $\sigma=J$. Each curve was obtained after averaging over $10^{6}$ realizations of disorder. The insets show enlarged views of the spectra within the small boxes.

absorption spectra are still featureless and no signatures of the WSL are detected. The corresponding fluorescence decay is shown in Fig. 4, where we observe that the decay is nonexponential. At a longer time, the decay is slower during an increase in the magnitude of the correlation, similar to what was found in the absence of bias (see Fig. 2).

In Fig. 5, we plotted the absorption spectra calculated for a high value of the bias, $U=0.5 \mathrm{~J}$. In the upper panel we observe that, at $\alpha<\alpha_{c}$, the spectrum remains structureless. However, in the lower panel, we see that for strong correlations in disorder, when $\alpha>\alpha_{c}$, the spectrum presents a periodic pattern that is not masked by the stochastic disorder fluctuations (see the inset in the lower panel). Most important, the period of the modulation is exactly equal to $U$ $=0.5 \mathrm{~J}$, which results from the occurrence of the WSL in the energy spectrum of the system.

As mentioned above, long-range correlations in disorder strongly affect the optical response of the system. We now present evidence that they also have remarkable impact on the fluorescence decay. More important, we will show that the peculiarities found in the fluorescence decay can be related to the occurrence of the WSL. To this end, we numerically solve Eqs. (2) and (5) to obtain the time dependence of the fluorescence when $U \neq 0$. Figure 6 shows the fluorescence decay after pulse excitation as a function of $\gamma t$ for various set parameters and $W_{0}=\gamma$. The lattice size is $N$ $=250$, the bias is $U=0.5 \mathrm{~J}$, and the results comprise the aver-

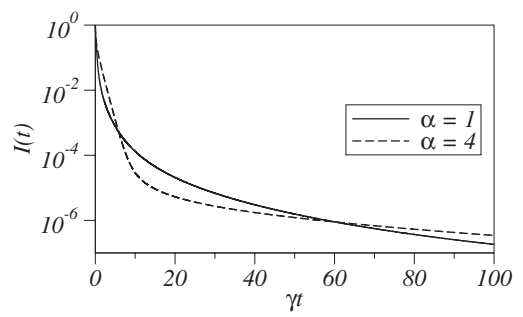

FIG. 4. Time dependence of the fluorescence decay after pulse excitation in weakly $(\alpha=1)$ and strongly $(\alpha=4)$ correlated 1D lattices when $U=0.05 J$ and $\sigma=J$. The lattice size is $N=250$ and the results comprise the average over 200 realizations of the disorder.

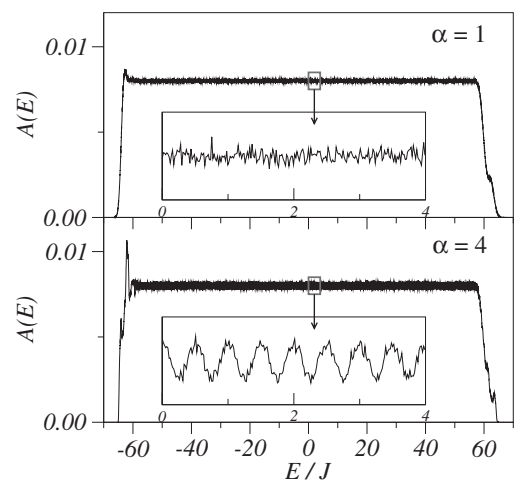

FIG. 5. Absorption spectra of biased chains $(U=0.5 J)$ with $N$ $=250$ sites calculated for two values of the correlation exponent $\alpha$, shown in the plot. The magnitude of disorder is $\sigma=J$. Each curve was obtained after averaging over $10^{6}$ realizations of disorder. The insets show enlarged views of the spectra within the small boxes.

age over 200 realizations of the disorder. We observe that the fluorescence decay is still nonexponential when $\alpha<\alpha_{c}$. However, the decay is exponential in the opposite limit, when $\alpha>\alpha_{c}$. It is to be noted that the system size $N$ should be sufficiently large enough to yield the power-law spectral density $S(k) \propto k^{-\alpha}$. If $N$ is inadequately small, $S(k)$ would not obey a power law. To ascertain whether the results mentioned above can be attributed to long-range spatial correlations, we also studied larger system sizes. Figure 6 also shows the fluorescence decay when $N=1000$; the results comprise the average over 50 realizations of the disorder. The nonexponential and exponential decays for weakly and strongly correlated disorder, respectively, are clearly observed (see Sec. VI below for a detailed discussion of finitesize effects).

In Ref. 14, we claimed that the WSL can be resolved even if correlations are weak. We pointed out that the condition for the WSL to appear in the absorption spectrum is $\sigma$ $>\sqrt{U J}$ in the weakly correlated case. We calculated the absorption spectrum line shape for magnitudes of disorder $\sigma$ $=0.2 \mathrm{~J}$ and bias $U=0.5 \mathrm{~J}$, when this inequality holds. The results obtained for $\alpha=1$ and $\alpha=4$ are depicted in Fig. 7 (upper and lower panels, respectively). One observes that the absorption spectrum shows a resolved WSL structure for both

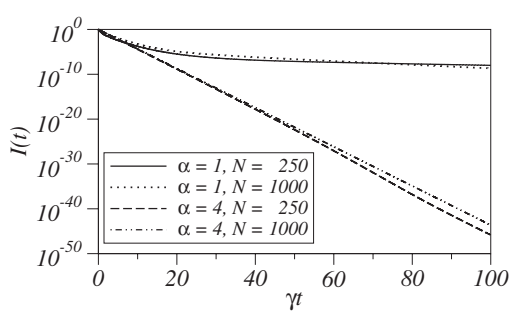

FIG. 6. Time dependence of the fluorescence decay after pulse excitation in weakly $(\alpha=1)$ and strongly $(\alpha=4)$ correlated 1D lattices when $U=0.5 J$ and $\sigma=J$. The lattice size is $N=250$ and the results comprise the average over 200 realizations of the disorder. For comparison, the results for larger systems $(N=1000)$ are also shown, comprising averages over 50 realizations of the disorder. 


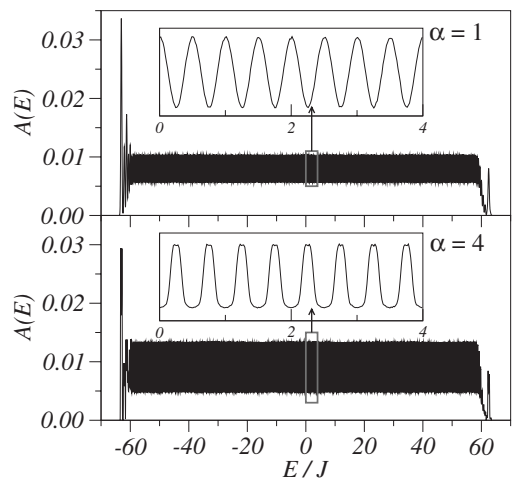

FIG. 7. Absorption spectra of biased chains $(U=0.5 J)$ with $N$ $=250$ sites calculated for two values of the correlation exponent $\alpha$, shown in the plot. The magnitude of disorder is $\sigma=0.2 \mathrm{~J}$. Each curve was obtained after averaging over $10^{6}$ realizations of disorder. The insets show enlarged views of the spectra within the small boxes.

values of $\alpha$. Figure 8 indicates that the fluorescence exponentially decays with time when $\alpha>\alpha_{c}$ and biexponentially when $\alpha<\alpha_{c}$.

The results shown in Figs. 6 and 8 provide compelling evidence that the occurrence of the WSL in the energy spectrum may result in an exponential decay of the fluorescence. In the upper panel of Fig. 5, we observed the absence of WSL signatures in the optical absorption spectrum when $\alpha$ $<\alpha_{c}$ and the magnitude of disorder $\sigma$ is large. Simultaneously, the fluorescence decay is nonexponential, as shown in Figs. 6 and 8. This behavior can be understood from the fact that when $\sigma$ is large and correlations are weak $\left(\alpha<\alpha_{c}\right)$, the oscillator strength depends on the energy state, similar to what is found in the absence of bias (see Sec. IV). This finally leads to a nonexponential decay of the fluorescence. However, when correlations are strong, $\alpha>\alpha_{c}$, or the magnitude of disorder $\sigma$ is smaller than $\sqrt{U J}$, the occurrence of the WSL ladder is accompanied by an exponential decay of the fluorescence, as shown in Fig. 6. In this case, localization by the bias $U$ prevails over localization by disorder. When the WSL arises, the shape of the eigenfunction is almost the same for all states, except for a trivial space shift and excluding finite-size effects (see Fig. 9). Consequently, the oscillator strength of all states has the same value, yielding a single exponential in the decay law of the fluorescence.

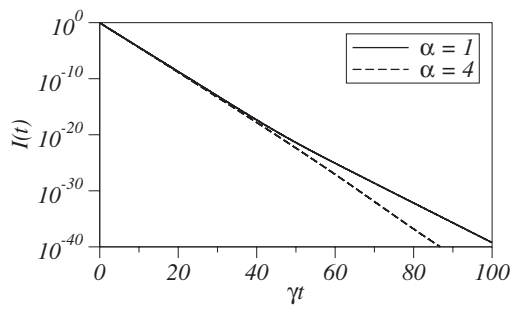

FIG. 8. Time dependence of the fluorescence decay after pulse excitation in weakly $(\alpha=1)$ and strongly $(\alpha=4)$ correlated 1D lattices when $U=0.5 \mathrm{~J}$ and $\sigma=0.2 \mathrm{~J}$. The lattice size is $N=250$ and the results comprise the average over 200 realizations of the disorder.

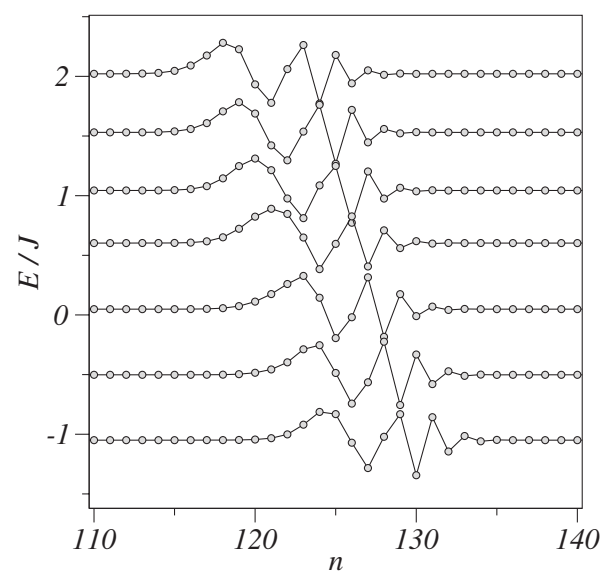

FIG. 9. A subset of eigenstates for a typical realization of the random energy potential when $U=0.5$ for a lattice of size $N=250$, magnitude of disorder $\sigma=0.2 \mathrm{~J}$, and correlation exponent $\alpha=1$. The baseline indicates the energy of the eigenstate.

\section{FINITE-SIZE EFFECTS}

The peculiarities observed at the low- and high-energy sides of the absorption spectra shown in Figs. 5 and 7 are associated with finite-size effects. ${ }^{14}$ The levels of these regions of the energy spectrum are formed by the states localized close to the system ends. Because of that, the corresponding eigenfunctions differ from those at the band center. As a consequence, not all eigenstates exactly present the same value of the oscillator strength and a slight deviation from the single exponential decay of the fluorescence can be detected.

To understand the relevance of finite-size effects, we focus on the biased, disorder-free lattice. In the thermodynamic limit $(N \rightarrow \infty)$, the normalized eigenstates of the Hamiltonian (2) with $\sigma=0$ are expressed in terms of the Bessel functions as $\psi_{\mu, n}=J_{n-\mu}(2 J / U)$ (see Ref. 24). The corresponding eigenenergies are $E_{\mu}=\mu U$, with $\mu$ being an integer (i.e., the WSL structure). The oscillator strength can also be exactly calculated in this limiting case $f_{\mu}=1$, which takes the same value for all states, as expected. Remarkably, the oscillator strength also becomes independent of the bias $U$ in large disorder-free systems. From Eq. (6), we conclude that the normalized fluorescence intensity is nothing but the survival probability $I(t)=\sum_{\mu=1}^{N} P_{\mu}(t)$ in this case. Summing over all states in Eq. (5), we obtain $\dot{I}(t)=-\gamma I(t)$, leading to $I(t)$ $=\exp (-\gamma t)$. Consequently, the fluorescence decay in biased disorder-free lattices is the same as in the isolated monomer (i.e., single exponential with decay time $1 / \gamma$ ) when the system is large enough.

As mentioned in the preceding paragraph, when $N \rightarrow \infty$, the occurrence of the WSL in the energy spectrum $\left(E_{\mu}\right.$ $=\mu U)$ in disorder-free systems leads to an exponential decay of the fluorescence. The decay time is found to be the same as in the single monomer and is, consequently, independent of the bias. We have performed simulations in a finite system when $\sigma=0$ to obtain the decay time $\tau_{\infty}$ from the fluorescence intensity curves when $t \rightarrow \infty$. Results are shown in Fig. 10, where we observe that $\gamma \tau_{\infty}$ approaches the theoretical limit 


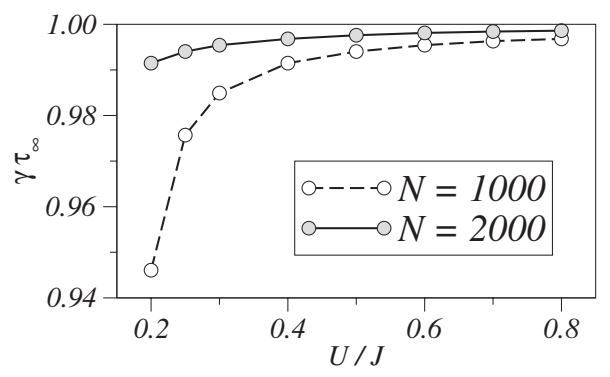

FIG. 10. Bias dependence of $\gamma \tau_{\infty}$ for different system sizes when $\sigma=0$.

$\left(\gamma \tau_{\infty}=1\right)$ during an increase in both the system size and the bias. The localization length $L_{U}$, due to the presence of the bias, decreases when $U$ increases, leading to smaller finitesize effects.

Finite-size effects are also relevant in a biased lattice with long-range-correlated disorder. In Fig. 11, we observe a trend similar to that found in disorder-free lattices. The main difference is related to the value of $\tau_{\infty}$, which is slightly lower than that in disorder-free lattices of the same size.

\section{CONCLUSIONS}

We numerically studied the intraband exciton relaxation in a $1 \mathrm{D}$ disordered lattice, which is subjected to a linear bias of magnitude $U$. The random site potential presents a powerlaw spectral density $S(k) \sim 1 / k^{\alpha}$, which gives rise to longrange correlations in site energies. Exciton transport is considered as an incoherent hopping over the eigenstates of the static lattice, which arises when the coupling to vibrations is taken into account. The dynamics of the intraband relaxation is monitored by means of the fluorescence decay after broadband pulse excitation.

Fluorescence decay in the unbiased lattice $(U=0)$ was found to be nonexponential in both weak $\left(\alpha<\alpha_{c}=2\right)$ and strong $\left(\alpha>\alpha_{c}=2\right)$ correlation limits. This time dependence reflects the existence of many decay channels in the system with different decay times. In other words, the wave functions vary from state to state and, consequently, the distribution of the oscillator strengths is rather broad. We found a slowdown of the fluorescence decay when $\alpha>\alpha_{c}$, which we

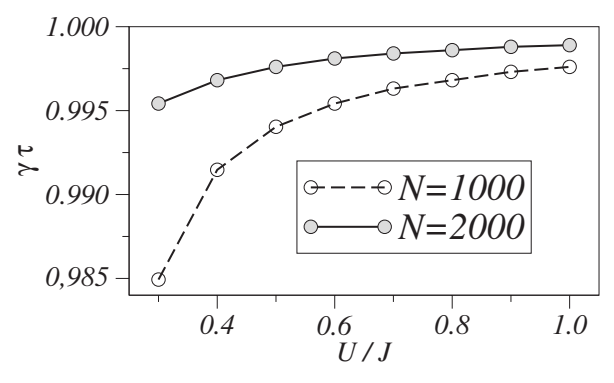

FIG. 11. Bias dependence of $\gamma \tau_{\infty}$ for different system sizes, when $\sigma=J$ and. The results comprise averages over 50 realizations of disorder.

relate to the peculiar level structure of level in this case: there exist dark states below those states with a high oscillator strength located deep in the band (such as that labeled 3 in Fig. 1).

At a moderate bias, the absorption spectrum broadens as compared to the unbiased case, but no signatures of the WSL are found. The structureless spectrum is accompanied by a nonexponential decay of the fluorescence. However, when the magnitude of the bias is further increased, a periodic pattern is found to build up at the center of the (already wide) absorption band when $\alpha>\alpha_{c}$. Its period is equal to $U$, which is the same as that for the WSL in an ideal lattice, and is independent of the system size $N$. Simultaneously, the fluorescence decay is approximately described by a single exponential. Finally, when the parameter $\sqrt{U J}$ exceeds the magnitude of disorder $\sigma$, the WSL in the absorption spectrum and the exponential decay of the fluorescence appear, irrespective of the value of the correlation exponent $\alpha$. The single exponential decay of the fluorescence is a direct consequence of the localization by the bias, since all states approximately carry the same value of the oscillator strength. Deviations from perfect exponential decay were mainly related not only to stochastic fluctuations of the disorder but also to finite-size effects.

\section{ACKNOWLEDGMENTS}

The authors thank V. A. Malyshev and Yu. A. Kosevich for helpful conversations. This work was supported by MEC (Project MOSAICO).
${ }^{1}$ M. Paczuski, S. Maslov, and P. Bak, Phys. Rev. E 53, 414 (1996).

${ }^{2}$ S. Havlin, S. V. Buldyrev, A. Bunde, A. L. Goldberger, P. Ch. Ivanov, C.-K. Peng, and H. E. Stanley, Physica A 273, 46 (1999)

${ }^{3}$ P. Carpena, P. Bernaola-Galván, P. Ch. Ivanov, and H. E. Stanley, Nature (London) 418, 955 (2002); 421, 764 (2003).

${ }^{4}$ H. Yamada, Phys. Lett. A 332, 65 (2004); Int. J. Mod. Phys. B 18, 1697 (2004); Phys. Rev. B 69, 014205 (2004).

${ }^{5}$ E. L. Albuquerque, M. S. Vasconcelos, M. L. Lyra, and F. A. B. F. de Moura, Phys. Rev. E 71, 021910 (2005).
${ }^{6}$ S. Roche, D. Bicout, and E. Maciá, Phys. Rev. Lett. 92, 109901 (2004).

${ }^{7}$ F. A. B. F. de Moura and M. L. Lyra, Phys. Rev. Lett. 81, 3735 (1998).

${ }^{8}$ F. M. Izrailev and A. A. Krokhin, Phys. Rev. Lett. 82, 4062 (1999).

${ }^{9}$ G.-P. Zhang and S.-J. Xiong, Eur. Phys. J. B 29, 491 (2002).

${ }^{10}$ H. Shima, T. Nomura, and T. Nakayama, Phys. Rev. B 70, 075116 (2004).

${ }^{11}$ F. Bloch, Z. Phys. 52, 555 (1928).

${ }^{12}$ F. Domínguez-Adame, V. A. Malyshev, F. A. B. F. de Moura, 
and M. L. Lyra, Phys. Rev. Lett. 91, 197402 (2003).

${ }^{13}$ G. H. Wannier, Phys. Rev. 117, 432 (1960).

${ }^{14}$ E. Díaz, F. Domínguez-Adame, Yu. A. Kosevich, and V. A. Malyshev, Phys. Rev. B 73, 174210 (2006).

${ }^{15}$ D. J. Heijs, V. A. Malyshev, and J. Knoester, J. Chem. Phys. 121, 4884 (2004).

${ }^{16}$ M. Bednarz, V. A. Malyshev, and J. Knoester, J. Chem. Phys. 117, 6200 (2002).

${ }^{17}$ M. Shimizu, S. Suto, and T. Goto, J. Chem. Phys. 114, 2775 (2001).

${ }^{18}$ M. Bednarz, V. A. Malyshev, J. P. Lemaistre, and J. Knoester, J. Lumin. 94-95, 271 (2001).
${ }^{19}$ A. V. Malyshev, V. A. Malyshev, and F. Domínguez-Adame, Chem. Phys. Lett. 371, 417 (2003).

${ }^{20}$ A. V. Malyshev, V. A. Malyshev, and F. Domínguez-Adame, J. Phys. Chem. 107, 4418 (2003).

${ }^{21}$ E. E. Méndez, F. Agulló-Rueda, and J. M. Hong, Phys. Rev. Lett. 60, 2426 (1988).

${ }^{22}$ F. Agulló-Rueda, E. E. Méndez, and J. M. Hong, Phys. Rev. B 40, 1357 (1989).

${ }^{23}$ M. K. Saker, D. M. Whittaker, M. S. Skolnick, M. T. Emeny, and C. R. Whitehouse, Phys. Rev. B 43, 4945 (1991).

${ }^{24}$ H. Fukuyama, R. A. Bari, and H. C. Fogedby, Phys. Rev. B 8, 5579 (1973). 